\title{
复杂多态系统的区间值模糊贝叶斯网络建模与 分析
}

\author{
米金华, 李彦锋*, 彭卫文, 黄洪钟 \\ 电子科技大学系统可靠性与安全性研究中心, 成都 611731 \\ *联系人, E-mail: yanfengli@uestc.edu.cn
}

收稿日期: 2016-12-16; 接受日期: 2017-02-07; 网络出版日期: 2017-07-28

国家自然科学基金(编号: 51405065)、中央高校基本科研业务费专项资金(编号: ZYGX2014Z010)和机械传动国家重点实验室开放基金(编 号: SKLMT-KFKT-201601)资助项目

\begin{abstract}
摘要随着现代工程系统结构复杂度的增加以及运行环境的复杂化, 传统的可靠性分析与评估技术已不能 满足现代工程系统需求。针对复杂多态系统可靠性分析与评估的需求, 本文从部件状态分析、系统结构分析入 手, 对存在认知不确定性同时考虑失效相关性的复杂多态系统进行可靠性建模与分析. 由于现代复杂多态系统 信息输入及系统故障影响因素的多样性, 以及系统冗余, 使得不确定性和共因失效对复杂多态系统可靠性分析 和评估至关重要. 本文借助贝叶斯网络对多态系统的图形表达和推理优势, 采用模糊理论中区间值三角模糊数 对部件概率进行描述; 同时利用 $\beta$ 因子模型对共因失效进行量化, 再将其以新增共因节点的方式实现共因失效 的贝叶斯网络建模, 从而提出基于区间值模糊贝叶斯网络的复杂多态系统可靠性分析方法. 最后, 将基于区间 值模糊多态贝叶斯网络对具有共因失效的多态卫星天线双轴定位机构传动系统进行可靠性分析. 通过对实际 工程系统的分析, 表明本文建立的多种因素影响下复杂多态系统的可靠性建模和分析方法, 进一步完善了复 杂系统可靠性分析理论与方法.
\end{abstract}

关键词认知不确定性, 多态系统, 区间值模糊概率, 贝叶斯网络, 共因失效, 可靠性建模

PACS: 47.27.-i, 47.27.Eq, 47.27.Nz, 47.40.Ki, 47.85.Gj

\section{1 引言}

在传统系统可靠性研究中, 常将事件发生概率视 为已知. 但是在实际工程中, 由于对系统认知不完全, 不宜采用传统概率方法对系统进行可靠性建模与分析.

系统的不确定性也是复杂系统的重要特性. 通常, 不确定性可分为两类: 随机不确定性和认知不确定性.
随机不确定性也称客观不确定性, 来源于系统固有偶 然性或变异性, 是不可避免的. 随机不确定性的描述 和传播常用概率论方法来处理. 认知不确定性是由于 知识的不完备以及数据的缺乏造成的, 也称主观不确 定性 ${ }^{[1,2]}$. 由于系统复杂、试验样本数有限以及数据不 足, 通常不能得到系统状态性能水平和状态概率的精 确值, 但可用语言形式表示系统状态或概率的上下限.

引用格式: 米金华, 李彦锋,彭卫文, 等. 复杂多态系统的区间值模糊贝叶斯网络建模与分析. 中国科学: 物理学力学 天文学, 2018, 48: 014604 Mi J H, Li Y F, Peng W W, et al. Reliability modeling and analysis of complex multi-state system based on interval fuzzy Bayesian network (in Chinese). Sci Sin-Phys Mech Astron, 2018, 48: 014604, doi: 10.1360/SSPMA2016-00521 
此时, 基于概率的方法不再适用, 而非概率方法如证 据理论、模糊理论、概率盒、区间理论、随机集理 论、信息差理论、可能性理论、贝叶斯方法等 ${ }^{[3-10]}$, 已 被提出并发展用于复杂系统的不确定性分析.

贝叶斯网络(Bayesian Network, BN)基于一套定义 完整的概率推理理论, 能够表达随机变量之间复杂的 相关关系, 目前已被应用于大量实际工程中, 尤其是 相关性评估、风险维修性分析等. 对于实际工程系统 表现出的多状态特性, 静态贝叶斯网络已经被用来进 行多态系统的可靠性评估 ${ }^{[11,12]}$. 之后, 考虑系统的动态 特性, 许多学者研究了动态故障树与动态贝叶斯网络 的关系, 通过将动态故障树转换为动态贝叶斯网络, 实现动态系统可靠性建模和评估 ${ }^{[13,14]}$. 贝叶斯网络推 理基于精确的节点概率描述, 但对于存在认知不确定 性或模糊性的多态系统, 此时, 如何应用贝叶斯网络 实现此类复杂多态系统的可靠性分析是一个值得研 究的问题.

随着现代工程系统复杂度及冗余度的增加, 部件 的独立失效对系统失效的贡献逐渐减小, 但多个部件 相关失效而引起的系统失效事件却呈增长趋势. 复杂 系统可靠性分析中, 相关失效是造成随机故障的主要 因素. 共因失效(Common Cause Failure, CCF) 已成为系 统可靠性分析中的一个重要问题. 对于复杂系统中广 泛存在的共因失效问题, 目前已发展了较多的对系统 共因失效进行表征的参数模型, 如 $\alpha$ 因子模型、 $\beta$ 因子 模型、基本参数模型(Basic Parameter, BP) ${ }^{[15]}$ 等. 然而, 在实际工程中, 对于大型、超大型复杂系统, 往往不 具备系统级可靠性试验的条件, 同时能够获得的部件 级可靠性试验数据也相当有限. 此时, 将无法得到足 够的试验数据来实现 $\alpha$ 因子模型的参数估计, 这已成 为制约 $\alpha$ 因子模型广泛使用的一大难题. 因此, 鉴于 $\beta$ 因子模型具有的简单以及适用性广等特点, 本文采用 $\beta$ 因子模型对复杂系统中的共因失效进行量化.

本文主要分为以下 3 个部分, 首先对模糊多态贝 叶斯网络方法进行详细介绍. 当考虑系统中模糊不确 定性时, 采用区间值三角模糊数对节点的模糊信息进 行表述, 给出区间值模糊多态贝叶斯网络的定义以及 区间值模糊数的归一化处理方法. 然后将系统中存在 的共因失效通过显式建模方法以新增共因节点的方 式融入贝叶斯网络建模的程序中, 并给出最常用的共 因失效量化的 $\beta$ 因子参数模型. 最后, 将基于区间值模
糊多态贝叶斯网络对具有共因失效的多态卫星天线 双轴定位机构传动系统进行可靠性分析.

\section{2 考虑认知不确定性的贝叶斯网络建模}

\section{1 模糊多态贝叶斯网络}

为了表征由于对系统认知不足及信息有限等原 因造成的主观不确定性对系统可靠性的影响,一些学 者提出将贝叶斯网络的连续节点变量进一步推广为 模糊节点变量 ${ }^{[16]}$. 当贝叶斯网络节点的故障概率难以 用精确值表示时, 采用模糊子集来表示节点的故障概 率. 为了便于运算, 本节仅采用三角模糊数对节点的 模糊故障概率进行描述.

定义 1: 三角模糊数. 若模糊数 $\widetilde{p}=\left(p^{l}, p^{m}, p^{u}\right)$, $p^{l}$ 和 $p^{u}$ 分别表示 $\widetilde{p}$ 所支撑的上下界, 且 $0<p^{l} \leq p^{m} \leq p^{u}, p^{m}$ 表 示 $\widetilde{p}$ 的中值, 则称 $\widetilde{p}$ 为一个三角模糊数, 其隶属函数 如图1所示并可表示为

$\mu_{\tilde{p}}(p)=\left\{\begin{array}{cc}0, & 0<p<p^{l}, \\ \frac{p-p^{l}}{p^{m}-p^{l}}, & p^{l} \leq p \leq p^{m}, \\ \frac{p^{u}-p}{p^{u}-p^{m}}, & p^{m} \leq p \leq p^{u}, \\ 0, & p^{u}<p .\end{array}\right.$

三角模糊数的加法、乘法和除法等基本运算法则 可参考文献[17-19]. 对于三角模糊数 $\widetilde{p}=\left(p^{l}, p^{m}, p^{u}\right)$, 其上下界均值可用下式来计算:

$$
\bar{M}(\widetilde{p})=2 \int_{0}^{1} \beta\left[p^{l}+(1-\beta) p^{u}\right] \mathrm{d} \beta,
$$

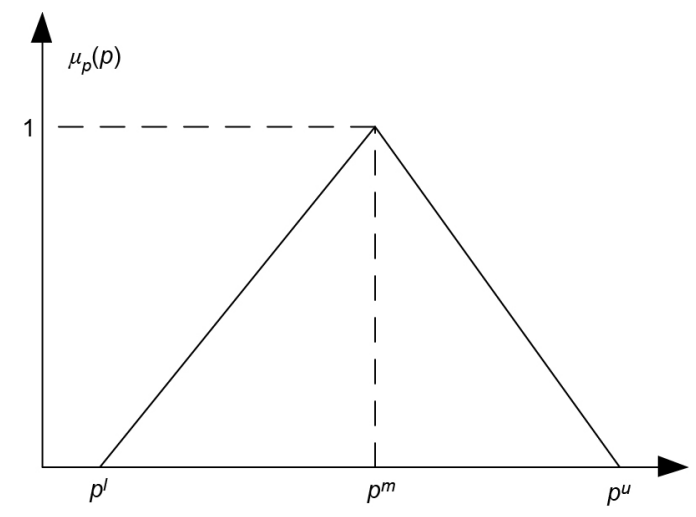

图 1 三角模糊数 $\widetilde{p}$ 的隶属函数

Figure 1 The membership function of triangular fuzzy number $\tilde{p}$. 
$\underline{M}(\widetilde{p})=2 \int_{0}^{1} \alpha\left[p^{l}-(1-\beta) p^{m}\right] \mathrm{d} \alpha$,

式中, 任意 $\alpha, \beta \in[0,1]$.

对于具有有限个节点的贝叶斯网络, 节点 的集合为 $\mathbf{X}=\left\{x_{1}, x_{2}, \ldots, x_{i}, \ldots, x_{n}\right\}(i=1,2, \ldots, n)$. 设 节点 $x_{i}$ 具有 $k_{i}$ 个模糊故障状态, 则其状态空间 为 $\boldsymbol{x}_{i}=\left\{x_{i, 1}, x_{i, 2}, \cdots, x_{i, j}, \cdots, x_{i, k_{i}}\right\}\left(j=1,2, \ldots, k_{i}\right)$. 设节点 $x_{i}$ 处于故障状态 $x_{i, k_{i}}$ 的故障概率是一个模糊子集, 且 $p^{m}-p^{l}$ 和 $p^{u}-p^{m}$ 分别为模糊子集的左右模糊区间, 取值 越大表示模糊化程度越强. $\mu_{i}{ }^{j}$ 为节点 $x_{i}$ 在模糊故障状 态 $j$ 时的隶属度, 表征节点故障信息的模糊不确定性 程度, 即节点故障概率的模糊性, 且有 $\sum_{j=1}^{k_{i}} \mu_{i}{ }^{j}=1$.

根据三角模糊数的定义, 每一个三角模糊数都有 一个非模糊数与之相对应, 找到能够最大程度表征此 模糊数的一个数值的过程叫作去模糊, 通常也叫做解 模糊. 目前, 去模糊的方法有很多, 包括均值面积法、 重心法、积分值法等 ${ }^{[20,21]}$.

利用专家知识以及实际经验对贝叶斯网络的条 件概率表进行重构, 从而使得传统贝叶斯网络不能表 达的部件故障逻辑关系、不确定性及多态特性得到 了体现. 因此, 利用贝叶斯网络处理变量间的多态性 时, 可选用不同的状态取值来表征节点的不同故障状 态, 只需调整相应节点的 CPT即可. 图 2 为一个简单的 具有三状态节点的模糊贝叶斯网络及其CPT.

图2中, 节点 $y$ 处于各个状态的概率值 $\widetilde{p}_{i, j}(1 \leq i \leq m$, $1 \leq j \leq 3)$ 为模糊数. 图 2 的条件概率表中, 各节点的故 障状态可用其所对应的变量符号取值 ( $k=0,1,2$ 等) 表 示. 贝叶斯网络节点间的故障逻辑关系可用条件概率 $P\left(y=k \mid x_{1}, x_{2}\right)$ 来表达, 从而节点间的故障逻辑关系将转 化为模糊贝叶斯网络中对应节点的 CPT. 此外, 通过改 变CPT中的条件概率可实现系统故障机理变化的表征.

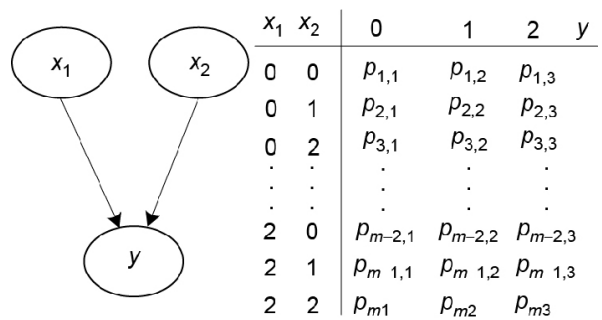

图 2 多态模糊贝叶斯网络及CPT

Figure 2 The multi-state fuzzy BN and its CPT.
将上例中三节点模糊多态贝叶斯网络作进一步拓展, 可得到具有 $n$ 个父节点 $\left(x_{1}, x_{2}, \ldots, x_{n}\right)$ 的节点 $y_{i}$ 的 $\mathrm{CPT}^{[22]}$.

由故障树转化而来的贝叶斯网络的根节点变量 为 $x_{i}(i=1,2, \ldots, n)$, 中间节点变量为 $y_{j}(j=1,2, \ldots, m)$, 叶节 点变量为 $T_{v}(v=1,2, \ldots, l)$, 采用模糊数 $x_{i}\left(k_{i}\right), y_{j}\left(k_{j}\right)$ 和 $T_{v}$ 分 别描述对应节点的故障状态, 当根节点故障状态的模 糊概率为 $\widetilde{P}\left(x_{i, k_{i}}\right)$ 时, 叶节点 $T$ 处于故障状态 $T_{v}$ 的模糊概 率为

$$
\begin{aligned}
& \widetilde{P}\left(T=T_{v}\right) \\
& =\sum_{x_{1}, \cdots, x_{n}, y_{1}, \cdots, v_{m}} \widetilde{P}\left(x_{1}, \cdots, x_{n}, y_{1}, \cdots, y_{m}, T=T_{v}\right) \\
& =\sum_{\pi(T)} \widetilde{P}\left(T=T_{v} \mid \pi(T)\right) \prod_{j=1}^{m} \sum_{\pi\left(y_{1}\right)} \widetilde{P}\left(y_{j} \mid \pi\left(y_{j}\right)\right) \prod_{i=1}^{n} \widetilde{P}\left(x_{i}^{k_{i}}\right) \\
& =\sum_{\pi(T)} \widetilde{P}\left(T=T_{v} \mid \pi(T)\right) \sum_{\pi\left(y_{1}\right)} \widetilde{P}\left(y_{1} \mid \pi\left(y_{1}\right)\right) \times \cdots \\
& \quad \times \sum_{\pi\left(v_{m}\right)} \widetilde{P}\left(y_{m} \mid \pi\left(y_{m}\right)\right) \times \cdots \times \widetilde{P}\left(x_{1, k_{1}}\right) \times \cdots \times \widetilde{P}\left(x_{1, k_{n}}\right),
\end{aligned}
$$

式中, $\pi(T)$ 表示节点 $T$ 的父节点集合, $\pi\left(y_{1}\right)$ 表示节点 $y_{1}$ 的所有父节点集合. 当节点 $x_{i}$ 的故障状态为 $x_{i, k_{i}}$ 时, 叶 节点 $T$ 处于故障状态 $T_{v}$ 的模糊条件概率为

$\widetilde{P}\left(T=T_{v} \mid x_{i}=x_{i, k_{i}}\right)=\frac{\widetilde{P}\left(T=T_{v}, x_{i}=x_{i, k_{i}}\right)}{\widetilde{P}\left(x_{i}=x_{i, k_{i}}\right)}$,

式中, $\widetilde{P}\left(T=T_{v}, x_{i}=x_{i, k_{i}}\right)$ 表示节点 $x_{i}$ 的故障状态为 $x_{i, k_{i}}$ 与 节点 $T$ 处于故障状态 $T_{v}$ 时的模糊联合概率. 利用贝叶 斯网络可在子节点发生故障已知的情况下计算其父 节点发生故障的后验概率, 即当叶节点 $T$ 故障状态为 $T_{v}$ 时, 根节点 $x_{i}$ 的状态为 $x_{i}^{{ }_{i}}$ 的后验概率为

$\widetilde{P}\left(x_{i}=x_{i, k_{i}} \mid T=T_{v}\right)=\frac{\widetilde{P}\left(T=T_{v}, x_{i}=x_{i, k_{i}}\right)}{\widetilde{P}\left(T=T_{v}\right)}$.

\section{2 区间值模糊多态贝叶斯网络}

当三角模糊数 $\tilde{p}$ 在任意点 $x$ 的隶属度无法用一个 精确的数值来表达时, 可以通过一定方法得到该点隶 属度的一个范围区间, 假设该范围可用 $\left[\underline{\mu}_{\tilde{p}}(x), \bar{\mu}_{\tilde{p}}(x)\right]$ 表 示. 此时可对三角模糊数进行区间推广, 构造出区间 值三角模糊数. 因此, 当贝叶斯网络节点故障模糊子集 的上限、下限难以用精确值表达时, 可引入区间变量 代替精确值, 构建区间模糊贝叶斯网络 ${ }^{[16,23]}$. 采用区间 值三角形隶属函数对节点的模糊故障概率进行描述. 
定义 2: 区间值三角模糊数. 令区间数 $\tilde{p}=[\underline{p}$, $\overline{\widetilde{p}}]$, 设其上下界 $\overline{\widetilde{p}}$ 和 $\underline{\tilde{p}}$ 均为模糊数, 即 $\underline{\tilde{p}}=\left(\underline{p}^{l}, p^{m}\right.$, $\left.\underline{p}^{u}\right), \overline{\widetilde{p}}=\left(\bar{p}^{l}, p^{m}, \bar{p}^{u}\right)$, 且参数满足 $0<\bar{p}^{l} \leq \underline{p}^{l} \leq p^{m} \leq$ $\underline{p}^{u} \leq \bar{p}^{u}$, 则称 $\tilde{p}=[\underline{\tilde{p}}, \overline{\widetilde{p}}]$ 为一个区间值三角模糊数, 本文将其表示为

$[\tilde{p}]=\left[\left(\underline{p}^{l}, p^{m}, \underline{p}^{u}\right),\left(\bar{p}^{l}, p^{m}, \bar{p}^{u}\right)\right]$,

式中, $\overline{\widetilde{p}}=\left(\bar{p}^{l}, p^{m}, \bar{p}^{u}\right)$ 表示区间上限三角模糊数, $\underline{\tilde{p}}=\left(\underline{p}^{l}, p^{m}, \underline{p}^{u}\right)$ 表示区间下限三角模糊数 ${ }^{[24]}$. 在一 些文献中, 也将区间值三角模糊数表示为 ${ }^{[17]}$

$[\widetilde{p}]=\left(\left[\bar{p}^{l}, \underline{p}^{l}\right], p^{m},\left[\underline{p}^{u}, \bar{p}^{u}\right]\right)$.

设某区间值三角模糊数如图 3 所示, 则其隶属函 数为

$\mu_{[\tilde{p}]}(p)=\left\{\begin{array}{cc}0, & 0 \leq p<\bar{p}^{l}, \\ {\left[\bar{p}^{l}, p\right],} & \bar{p}^{l} \leq p<\underline{p}^{l}, \\ {\left[1-\frac{p^{m}-p}{p^{m}-\underline{p}^{l}}, 1-\frac{p^{m}-p}{p^{m}-\bar{p}^{l}}\right],} & \underline{p}^{u}<p \leq p^{m}, \\ {\left[1-\frac{p-p^{m}}{\underline{p}^{u}-p^{m}}, 1-\frac{p-p^{m}}{\bar{p}^{u}-p^{m}}\right],} & p^{m}<p \leq \bar{p}^{u}, \\ {\left[\underline{p}^{u}, p\right],} & \underline{p}^{u} \leq p<\bar{p}^{u}, \\ 0, & \bar{p}^{u} \leq p<1 .\end{array}\right.$

对于非负区间值模糊数 $[\tilde{p}]_{1}=\left[\left(p_{1}^{l}, p_{1}{ }^{m}, \underline{p}_{1}^{u}\right),\left(\bar{p}_{1}^{l}\right.\right.$, $\left.\left.p_{1}{ }^{m}, \bar{p}_{1}^{u}\right)\right]$ 和 $[\tilde{p}]_{2}=\left[\left(\underline{p}_{2}^{l}, p_{2}^{m}, \underline{p}_{2}^{u}\right),\left(\bar{p}_{2}^{l}, p_{2}^{m}, \bar{p}_{2}^{u}\right)\right]$, 其基本

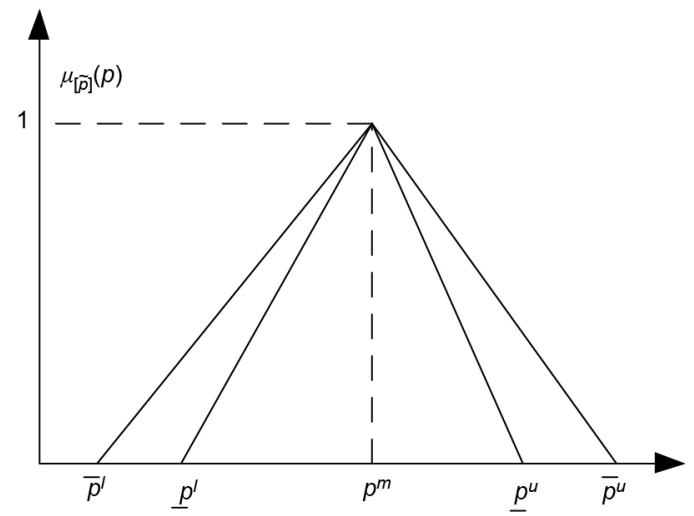

图 3 区间值三角模糊数隶属函数

Figure 3 The membership function of an interval triangular fuzzy number.
运算法则如下 ${ }^{[25]}$ :

$$
\begin{aligned}
{[\tilde{p}]_{1}+[\widetilde{p}]_{2}=} & {\left[\left(\bar{p}_{1}^{l}+\bar{p}_{2}^{l}, p_{1}^{m}+p_{2}^{m}, \bar{p}_{1}^{u}+\bar{p}_{2}^{u}\right),\right.} \\
& \left.\left(\underline{p}_{1}^{l}+\underline{p}_{2}^{l}, p_{1}^{m}+p_{2}^{m}, \underline{p}_{1}^{u}+\underline{p}_{2}^{u}\right)\right] \\
= & \left(\left[\bar{p}_{1}^{l}+\bar{p}_{2}^{l}, \underline{p}_{1}^{l}+\underline{p}_{2}^{l}\right], p_{1}^{m}+p_{2}^{m},\right. \\
& {\left.\left[\underline{p}_{1}^{u}+\underline{p}_{2}^{u}, \bar{p}_{1}^{u}+\bar{p}_{2}^{u}\right]\right), }
\end{aligned}
$$

$$
\begin{aligned}
{[\widetilde{p}]_{1} \times[\widetilde{p}]_{2}=} & {\left[\left(\bar{p}_{1}^{l} \cdot \bar{p}_{2}^{l}, p_{1}^{m} \cdot p_{2}^{m}, \bar{p}_{1}^{u} \cdot \bar{p}_{2}^{u}\right),\right.} \\
& \left.\left(\underline{p}_{1}^{l} \cdot \underline{p}_{2}^{l}, p_{1}^{m} \cdot p_{2}^{m}, \underline{p}_{1}^{u} \cdot \underline{p}_{2}^{u}\right)\right] \\
= & \left(\left[\bar{p}_{1}^{l} \cdot \bar{p}_{2}^{l}, \underline{p}_{1}^{l} \cdot \underline{p}_{2}^{l}\right], p_{1}^{m} \cdot p_{2}^{m},\right. \\
& {\left.\left[\underline{p}_{1}^{u} \cdot \underline{p}_{2}^{u}, \bar{p}_{1}^{u} \cdot \bar{p}_{2}^{u}\right]\right), }
\end{aligned}
$$

$$
\begin{aligned}
\frac{1}{[\widetilde{p}]_{1}} & =\left[\left(\frac{1}{\bar{p}_{1}^{u}}, \frac{1}{p_{1}{ }^{m}}, \frac{1}{\bar{p}_{1}^{l}}\right),\left(\frac{1}{\underline{p}_{1}^{u}}, \frac{1}{p_{1}^{m}}, \frac{1}{\underline{p}_{1}^{l}}\right)\right] \\
& =\left(\left[\frac{1}{\bar{p}_{1}^{u}}, \frac{1}{\underline{p}_{1}^{u}}\right], \frac{1}{p_{1}^{m}},\left[\frac{1}{\underline{p}_{1}^{l}}, \frac{1}{\bar{p}_{1}^{l}}\right]\right), \\
k[\widetilde{p}]_{1} & =\left[\left(k \bar{p}_{1}^{l}, k p_{1}^{m}, k \bar{p}_{1}^{u}\right),\left(k \underline{p}_{1}^{l}, k p_{1}^{m}, k \underline{p}_{1}^{u}\right)\right] \\
& =\left(\left[k \bar{p}_{1}^{l}, k \underline{p}_{1}^{l}\right], k p_{1}{ }^{m},\left[k \underline{p}_{1}^{u}, k \bar{p}_{1}^{u}\right]\right) .
\end{aligned}
$$

上述基本运算法则中涉及区间数的加法运算、 乘法运算和除法运算法则. 当区间数 $[x]$ 和 $[y]$ 均为正 区间数时, 其加法、乘法和除法运算法则可参考文 献[26,27].

定义 3: 对于区间值三角模糊数 $[\widetilde{p}]=\left[\left(\underline{p}^{l}, p^{m}\right.\right.$, $\left.\left.\underline{p}^{u}\right),\left(\bar{p}^{l}, p^{m}, \bar{p}^{u}\right)\right]$, 其上界均值 $\bar{M}([\tilde{p}])$ 和下界均 值 $\underline{M}([\tilde{p}])$ 分别为

$$
\begin{aligned}
\bar{M}([\tilde{p}])= & \int_{0}^{1} \alpha\left[\bar{p}^{u}+\left(p^{m}-\bar{p}^{u}\right) \alpha\right] \mathrm{d} \alpha \\
& +\int_{0}^{1} \beta\left[\underline{p}^{u}+\left(p^{m}-\underline{p}^{u}\right) \beta\right] \mathrm{d} \beta \\
= & \frac{\bar{p}^{u}+4 p^{m}+\underline{p}^{u}}{6}, \\
\underline{M}([\tilde{p}])= & \int_{0}^{1} \alpha\left[\bar{p}^{l}+\left(p^{m}-\bar{p}^{l}\right) \alpha\right] \mathrm{d} \alpha \\
& +\int_{0}^{1} \beta\left[\underline{p}^{l}+\left(p^{m}-\underline{p}^{l}\right) \beta\right] \mathrm{d} \beta \\
= & \frac{\bar{p}^{l}+4 p^{m}+\underline{p}^{l}}{6},
\end{aligned}
$$


式中, $\alpha, \beta \in[0,1]$. 依据式(14)和(15), 计算得到区间值 三角模糊数的区间均值为 $M([\widetilde{p}])=[\underline{M}([\widetilde{p}]), \bar{M}([\widetilde{p}])]$. 当根节点的所有故障状态区间值模糊概 率 $\widetilde{p}]\left(x_{i, k_{i}}\right)$ 已知时, 通过对式(4)进行拓展, 可得叶节点 $T$ 处于故障状态 $T_{v}$ 的区间值模糊概率为

$$
\begin{aligned}
& {[\widetilde{P}]\left(T=T_{v}\right) } \\
= & \sum_{x_{1}, \ldots, x_{n}, y_{1}, \ldots, y_{m}}[\widetilde{P}]\left(x_{1}, \ldots, x_{n}, y_{1}, \ldots, y_{m}, T=T_{v}\right) \\
= & \sum_{\pi(T)}[\widetilde{P}]\left(T=T_{v} \mid \pi(T)\right) \\
& \cdot \prod_{j=1}^{m} \sum_{\left.\pi / y_{j}\right)}[\widetilde{P}]\left(y_{j} \mid \pi\left(y_{j}\right)\right) \prod_{i=1}^{n}[\widetilde{P}]\left(x_{i, k_{i}}\right) \\
= & \sum_{\pi(T)}[\widetilde{P}]\left(T=T_{v} \mid \pi(T)\right) \sum_{\pi\left(y_{1}\right)}[\widetilde{P}]\left(y_{1} \mid \pi\left(y_{1}\right)\right) \\
& \times \ldots \times \sum_{\pi\left(y_{m}\right)}[\widetilde{P}]\left(y_{m} \mid \pi\left(y_{1}\right)\right) \\
& \times \ldots \times[\widetilde{P}]\left(x_{1, k_{1}}\right) \times \ldots \times[\widetilde{P}]\left(x_{n, k_{n}}\right),
\end{aligned}
$$

依据式(5), 当叶节点 $T$ 故障状态为 $T_{v}$ 时, 根节点 $x_{i}$ 的故 障状态 $x_{i, k_{i}}$ 的区间值模糊后验概率为

$[\widetilde{P}]\left(x_{i}=x_{i, k_{i}} \mid T=T_{v}\right)=\frac{[\widetilde{P}]\left(T=T_{v}, x_{i}=x_{i, k_{i}}\right)}{[\widetilde{P}]\left(T=T_{v}\right)}$,

式中, $[\widetilde{P}]\left(T=T_{v}, x_{i}=x_{i, k_{i}}\right)$ 为根节点 $x_{i}$ 处于故障状态 $x_{i, k_{i}}$, 叶节点 $T$ 处于故障状态 $T_{v}$ 的后验概率的区间值模糊子集. 取区间值模糊子集的重心值 $E\left[\left[\widetilde{P}\left(x_{i}=x_{i, k_{i}} \mid T=T_{v}\right)\right]\right.$ 为转 化的精确值, 则可实现对区间值模糊子集的精确化处 理 ${ }^{[16,23,28]}$.

\section{3 区间值三角模糊概率的归一化方法}

一般来讲, 概率的取值应处于区间 $[0,1]$ 内, 当对模 糊贝叶斯网络中间节点及叶节点进行模糊条件概率 推理时, 计算的结果存在超出 $[0,1]$ 区间的情况, 这是 不符合实际的, 此时可用如下的归一化方法对条件概 率数据进行处理 ${ }^{[29]}$. 设由 $N$ 个三角模糊数组成的一组 概率数据为 $\widetilde{p}_{i}=\left(p_{i}^{l}, p_{i}{ }^{m}, p_{i}{ }^{u}\right),(1 \leq i \leq N)$, 令

$$
t=\sup _{i=1, \cdots N}\left(\widetilde{p}_{i}\right)=\sup _{i=1, \cdots N}\left(p_{i}^{l}, p_{i}^{m}, p_{i}^{u}\right)=\sup _{i=1, \cdots N}\left(p_{i}^{u}\right)
$$

设 $t=p_{k}^{u}(1 \leq k \leq N)$, 对应于概率数据组中的第 $k$ 个模 糊数 $\widetilde{p}_{k}=\left(p_{k}^{l}, p_{k}^{m}, p_{k}^{u}\right)$ 的上界值, 定义归一化因子为

$$
w=\frac{1-p_{k}^{m}}{\max \left(1, p_{k}^{u}\right)-p_{k}^{m}} .
$$

应用归一化因子 $w$ 对三角模糊数 $\widetilde{p}_{i}$ 进行归一化处 理, 归一化后的第 $i$ 个模糊数据可以表示为

$$
\vec{p}_{i}=\left(p_{i}^{m}-w\left(p_{i}^{m}-p_{i}^{l}\right), p_{i}^{m}, p_{i}^{m}+w\left(p_{i}^{u}-p_{i}^{m}\right)\right) .
$$

由式(20)可得到一组归一化后的模糊概率.

对前文模糊数的归一化方法进行如下的拓展. 对于由 $N$ 个区间值三角模糊数组成的模糊概率数 据 $[\widetilde{p}]_{i}=\left(\left[\bar{p}_{i}^{l}, \underline{p}_{i}^{l}\right], p_{i}^{m},\left[\underline{p}_{i}^{u}, \bar{p}_{i}^{u}\right)\right), 1 \leq i \leq N$, 式(18)可进一步 拓展为

$$
\begin{aligned}
{[t] } & =\sup _{i=1, \cdots, N}\left([\widetilde{p}]_{i}\right)=\sup _{i=1, \ldots, N}\left(\left[\bar{p}_{i}^{l}, \underline{p}_{i}^{l}\right], p_{i}^{m},\left[\underline{p}_{i}{ }^{u}, \bar{p}_{i}^{u}\right]\right) \\
& =\sup _{i=1, \cdots, N}\left(\left[\underline{p}_{i}^{u}, \bar{p}_{i}^{u}\right]\right),
\end{aligned}
$$

式中, $\left[\underline{p}_{1}^{u}, \bar{p}_{1}^{u}\right], \ldots,\left[\underline{p}_{i}^{u}, \bar{p}_{i}^{u}\right], \ldots,\left[\underline{p}_{N}^{u}, \bar{p}_{N}^{u}\right]$ 表示由区间值 模糊数上界数据构成的一组区间数, 式(21)表示取这 一组区间数的上确界. 此时将涉及区间数的大小比较, 本节应用基于可能度的 NSG方法来处理.

对于区间数 $[p]_{i}^{u}=\left[\underline{p}_{i}^{u}, \bar{p}_{i}^{u}\right]$ 和 $[p]_{j}^{u}=\left[\underline{p}_{j}^{u}, \bar{p}_{j}^{u}\right]$, 日本 学者Nakahara等人 ${ }^{[27]}$ 提出了区间数大小比较的可能度 公式, 即

$$
\begin{aligned}
& P\left([p]_{i}^{u} \geq[p]_{j}^{u}\right) \\
& =\min \left\{\max \left\{\frac{\bar{p}_{i}^{u}-\underline{p}_{j}^{u}}{\left(\bar{p}_{i}^{u}-\underline{p}_{i}^{u}\right)+\left(\bar{p}_{j}^{u}-\underline{p}_{j}^{u}\right)}, 0\right\}, 1\right\},
\end{aligned}
$$

上式表示区间数 $[p]_{i}^{u}$ 以 $P\left([p]_{i}^{u} \geq[p]_{j}^{u}\right)$ 的可能度大于 $[p]_{j}^{u}$, $P\left([p]_{i}^{u} \geq[p]_{j}^{u}\right)>0.5$ 表示 $[p]_{i}^{u}$ 大于 $[p]_{j}^{u}$ 的可能性较大, 在 进行区间数大小比较时认定 $[p]_{i}^{u}>[p]_{j}^{u}$, 否则 $[p]_{i}^{u}<[p]_{j}^{u}$.

设 $[t]=[p]_{k}^{u}(1 \leq k \leq N)$, 其对应于概率数据组中第 $k$ 个区间值模糊数 $[\widetilde{p}]_{k}=\left(\left[\bar{p}_{k}^{l}, \underline{p}_{k}^{l}\right], p_{k}^{m},\left[\underline{p}_{k}^{u}, \bar{p}_{k}^{u}\right]\right)$ 的上界 值, 对式(19)进行改进得到如下新的归一化因子:

$[w]=\frac{1-p_{k}^{m}}{\max \left(1,[p]_{k}^{u}\right)-p_{k}^{m}}$,

式中, 新的归一化因子为一个区间数.下面对这一组 区间值三角模糊数进行归一化处理, 得到第 $i$ 个归一化 的区间值三角模糊数为 


$$
\begin{aligned}
{[\vec{p}]_{i}=} & \left(p_{i}^{m}-[w]\left(p_{i}^{m}-[p]_{i}^{l}\right),\right. \\
& \left.p_{i}^{m}, p_{i}^{m}+[w]\left([p]_{i}^{u}-p_{i}^{m}\right)\right) .
\end{aligned}
$$

式(23)和(24)中涉及的区间数的运算可用前面所 提的区间数运算规则来完成.

\section{3 系统可靠性的共因失效建模程序}

\section{1 共因失效贝叶斯网络建模}

运用贝叶斯网络来建立共因失效下系统可靠性 模型的关键是将共因失效部件的失效率 $\lambda_{t}$ 分解为独立 失效率 $\lambda_{I}$ 和共因失效率 $\lambda_{c}{ }^{[30]}$. 本节将通过以下几种典型 系统的共因失效建模来说明基于贝叶斯网络模型建 立系统可靠性共因失效模型的基本程序. 对于具有 $m$ 个状态的部件, 假定状态“0”代表部件处于完好状态, 状态“1”代表部件处于完全失效状态, 在状态“ 0 ”与状 态“1”之间有 $(m-2)$ 个状态, 这 $(m-2)$ 个状态表示部件处 于完好状态与完全失效状态之间不同的性能水平.

(1) 串联系统

串联系统是系统可靠性分析中最为简单, 也最 为常见的一种典型系统. 一个由 $n$ 个部件构成的串联 系统, 令 $R_{i}(t)$ 和 $\lambda_{i}(t)$ 分别表示部件 $i$ 的可靠度和失效率, $R_{s}(t)$ 表示系统的可靠度, 则串联系统数学模型为 ${ }^{[3]}$

$$
R_{s}(t)=\prod_{i=1}^{n} R_{i}(t)=\prod_{i=1}^{n} \exp \left(\int_{0}^{t} \lambda_{i}(t) \mathrm{d} t\right) .
$$

当两部件串联系统存在共因失效时, 其贝叶斯网络 模型如图4所示. 图中, $X_{i}(i=1,2)$ 是根节点, $D_{1}$ 和 $D_{2}$ 是中 间节点, $X_{1}$ 与 $C, X_{2}$ 与 $C$, 以及 $D_{1}$ 与 $D_{2}$ 之间都是串联关系.

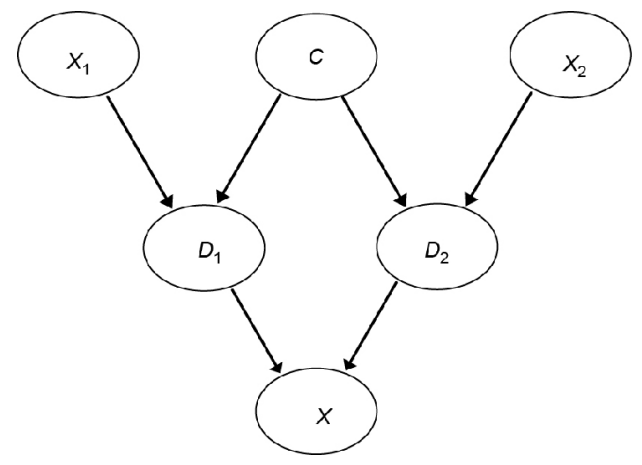

图 4 考虑共因失效的串联系统 $\mathrm{BN}$ 模型

Figure 4 The system BN model when considering CCF.
本文采用大写字母表示事件或贝叶斯网络中的 节点, 小写字母表示与大写字母相对应的变量. 则两 部件串联系统可靠度的数学表达式为 ${ }^{[32]}$

$$
\begin{aligned}
P(x=0)= & \sum_{x_{1}, x_{2}, c, d_{1}, d_{2}} P\left(x_{1}, x_{2}, c, d_{1}, d_{2}, x\right) \\
= & \sum_{d_{1}, d_{2}}\left\{P\left(x=0 \mid d_{1}, d_{2}\right)\right. \\
& \cdot \sum_{x_{1}, c}\left[P\left(d_{1}=0 \mid x_{1}, c\right) P\left(x_{1}\right) P(c)\right] \\
& \left.\cdot \sum_{x_{2}, c}\left[P\left(d_{2}=0 \mid x_{2}, c\right) P\left(x_{2}\right) P(c)\right]\right\} \\
= & P\left(x_{1}=0\right) P\left(x_{2}=0\right) P(c=0) .
\end{aligned}
$$

(2) 并联系统

两部件并联系统的贝叶斯网络模型与串联系统 基本相似, 其区别在于上面图4中的 $D_{1}$ 和 $D_{2}$ 由串联关 系变成了并联关系. 两部件并联系统可靠度的数学模 型表达如下:

$R_{s}(t)=1-\prod_{i=1}^{n}\left[1-R_{i}(t)\right]$.

当 $X_{1}=X_{2}$ 时, 对应的两部件为同一类部件, 则系统 可靠度的表达式为

$$
\begin{aligned}
P(x=0) & =\sum_{x_{1}, x_{2}, c, d_{1}, d_{2}} P\left(x_{1}, x_{2}, c, d_{1}, d_{2}, x\right) \\
& =2 P\left(x_{1}=0\right) P(c=0)-P^{2}\left(x_{1}=0\right) P(c=0) .
\end{aligned}
$$

\section{$3.2 \beta$ 因子参数模型}

直接精确测量共因失效事件的发生概率是非常 困难的, 因此, 现有研究通常采用参数化建模的方法 来量化共因失效发生概率, 如 $\alpha$ 因子方法、 $\beta$ 因子方法 等. 这些参数值一般由工程经验来确定, 或从现有相 关文献中获取, 本文将采用 $\beta$ 因子参数模型法来分析 传动系统的共因失效 ${ }^{[33]}$.

假设 $P_{t}$ 为部件的总失效概率, 将其分为独立失效 概率 $P_{\text {ind }}$ 和相关失效概率 $P_{\text {ccf. }}$ 假设部件服从指数分布, 用 $\lambda_{t}, \lambda_{\text {ind }}$ 和 $\lambda_{\mathrm{ccf}}$ 分别表示整个部件、独立部分和相关部 分的失效率, 参数 $\beta$ 被定义为相关失效的概率在总失 效概率中所占的比重 ${ }^{[34]}$, 即

$\beta=\frac{P_{\text {cff }}}{P_{t}}=\frac{P_{\text {cf }}}{P_{\text {ind }}+P_{\text {cof }}}=\frac{1-\exp \left(-\lambda_{\text {cof }} \cdot t\right)}{1-\exp \left(-\lambda_{t} \cdot t\right)}$ 


$$
=\frac{1-\exp \left(-\lambda_{\text {cof }} \cdot t\right)}{\left(1-\exp \left(-\lambda_{\text {ind }} \cdot t\right)\right)+\left(1-\exp \left(-\lambda_{\text {cef }} \cdot t\right)\right)} .
$$

$\beta$ 因子反映了相关部件对环境应力的敏感程度, 如物理和人因的影响. 如果相关部件对环境应力敏 感, $\beta$ 因子的取值将会较大. $\beta$ 因子的取值可直接通过 使用现场数据和专家经验获得. 一般来说, $\beta$ 因子取值 范围约为 $0-0.25, \beta=0$ 表示共因失效事件没有发生. 对 于硬件失效, 专家经验给出其失效的 $\beta$ 因子范围约为 0.001-0.10.

\section{4 实例分析}

随着航天技术的发展, 高可靠、长寿命逐渐成为 航天产品的普遍需求, 同时也成为了航天工业的最终 目标. 双轴定位机构被广泛应用于军事通信卫星、星 际探测卫星以及地球观测卫星等多种卫星系统 ${ }^{[35-37]}$. 作为卫星天线常用的控制机构, 双轴定位机构直接关 系着天线的指向精度, 并影响卫星发射和运行的可靠 性 ${ }^{[36]}$

\section{1 卫星天线双轴定位机构传动系统BN建模}

双轴定位机构是实现卫星在较大范围内旋转, 且 保持较高指向精度的重要部件. 根据统计, 双轴定位 机构失效概率相对较高, 对其开展可靠性分析具有非 常重要的意义. 从功能上讲, 双轴定位机构可划分为 两个子系统: 传动系统和控制系统, 本节仅对传动系 统进行研究. 传动系统通过调整俯仰轴和方位轴来 实现卫星天线的精确指向, 根据传动系统的结构和基 本工作原理, 传动系统的可靠性框图为串-并联结构, 如图5所示. 图中, 俯仰轴和方位轴均由步进电机、驱 动轴和谐波减速器串联连接而成. 本文将俯仰轴和方 位轴之间视为简单的并联连接关系, 也就是说, 其中 一个轴失效不会导致整个系统的失效.

由于指向机构的俯仰轴和方位轴是并联结构, 两 组部件的状态 ( $A_{1}$ 是俯仰轴的状态, $A_{2}$ 是方位轴的状态) 与系统状态 $(X)$ 之间的关系如下: 两组部件都失效会 导致整个传动系统失效; 如果一组部件失效, 另一组 部件部分失效, 系统处于部分失效状态; 只要两组部 件中任何一组完好工作, 系统则处于正常工作状态.

贝叶斯网络与可靠性框图在结构原理上具有相 似性, 因此贝叶斯网络模型可由可靠性框图模型映射
转化而得. 由图 5 可得如图6所示的传动系统贝叶斯网 络的拓扑结构. 根据系统失效机理分析, 两组部件可 视为具有 3 个状态的子系统. 步进电机和谐波减速器 都具有 3 个状态: 失效、部分失效和工作. 驱动轴只有 2 个状态: 失效和工作. 对于叶节点表示的系统事件 $X$ : $x=0$ 表示工作状态, $x=1$ 表示部分失效, $x=2$ 表示失效. 由 根节点 $B_{i 1}, B_{i 2}$ 与 $B_{i 3}(i=1,2)$ 的串联关系得到贝叶斯网络 中间节点 $A_{1}, A_{2}$ 的条件概率表如表 1所示. 中间节点 $A_{1}$ 和 $A_{2}$ 是并联关系, 从而得到叶节点 $X$ 的条件概率表如

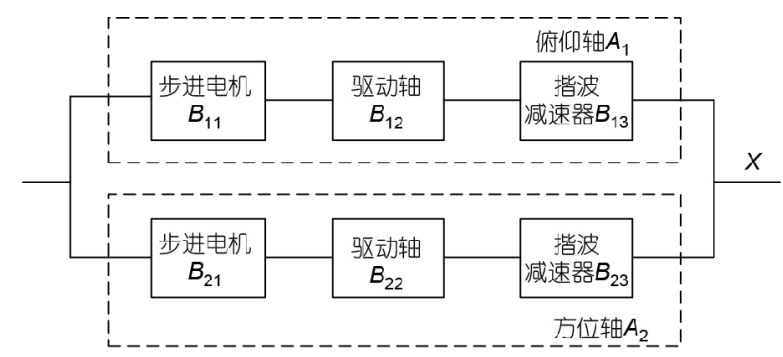

图 5 传动系统的可靠性框图

Figure 5 The reliability block diagram of transmission system.

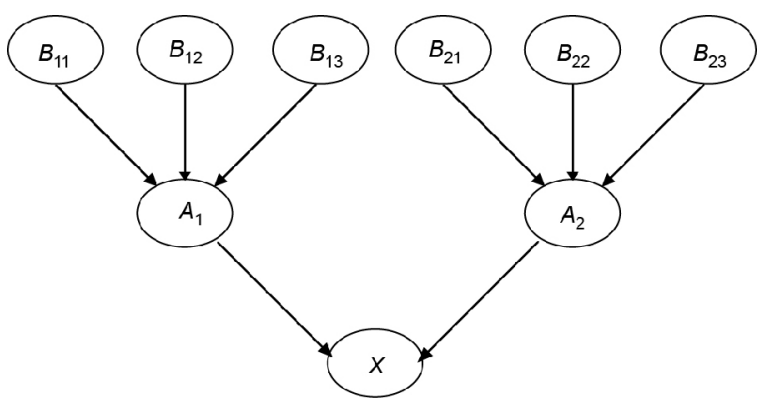

图 6 传动系统贝叶斯网络

Figure 6 BN model of transmission system.

表 1 叶节点 $X$ 的条件概率表

Table 1 The conditional probability table of leaf node $X$

\begin{tabular}{lllll}
\hline$a_{1}$ & $a_{2}$ & \multicolumn{3}{c}{$x$} \\
\cline { 3 - 5 } & 0 & 1 & 0 & 0 \\
0 & 1 & 1 & 0 & 0 \\
0 & 2 & 1 & 0 & 0 \\
1 & 0 & 1 & 0 & 0 \\
1 & 1 & 0 & 1 & 0 \\
1 & 2 & 0 & 1 & 0 \\
2 & 0 & 1 & 0 & 0 \\
2 & 1 & 0 & 1 & 0 \\
2 & 2 & 0 & 0 & 1 \\
\hline
\end{tabular}


表2所示.

由于卫星天线双轴定位机构系统复杂, 研制成本 高, 不可能对其进行大批量可靠性试验, 同时也不能获 得足够多的现场数据, 进而导致双轴定位机构存在数 据不足问题, 此时, 根节点状态分布概率需要根据相 关领域专家的经验来确定 ${ }^{[33,35,38]}$. 表2中给出了图6中 贝叶斯网络根节点在 $t=3000 \mathrm{~h}$ 时的区间三角模糊先验 概率分布 ${ }^{[35]}$.

\section{2 基于区间值模糊多态 BN的系统可靠性分析}

根据贝叶斯网络中节点之间的连接关系, 可较容 易地确定与系统和子系统对应的叶节点和中间节点的 边缘概率分布. 本节直接运用区间值三角模糊数的运 算规则进行贝叶斯网络推理计算, 依据式(26)和(28), 系统在各个状态下的概率为

$[\widetilde{p}](X=j)=\sum[\widetilde{p}]\left(b_{11}, b_{12}, b_{13}, a_{1}, b_{21}, b_{22}, b_{23}, a_{2}, x\right)$,

式中, $b_{i 1}, b_{i 3}, a_{1}, a_{2}, x \in\{0,1,2\}, b_{i 2} \in\{0,2\}, i=1,2, j=0,1$, 2. 基于式(30)与图6中的贝叶斯网络, 得到系统可靠度 的详细表达式为

$$
\begin{aligned}
& {[\widetilde{P}](X=0) } \\
= & \sum_{b_{11}, \cdots, b_{23}, a_{1}, a_{2}}[\widetilde{P}]\left(b_{11}, \cdots, b_{23}, a_{1}, a_{2}, x\right) \\
= & \sum_{a_{1}, a_{2}}[\widetilde{P}]\left(X=0 \mid a_{1}, a_{2}\right) \\
& \cdot \prod_{j=1}^{2}\left\{\sum_{b_{j 1}, b_{j 2}, b_{j 3}}[\widetilde{P}]\left(a_{j} \mid b_{j 1}, b_{j 2}, b_{j 3}\right) \prod_{i=1}^{3}[\widetilde{P}]\left(b_{j, i}\right)\right\} \\
= & \sum_{a_{1}, a_{2}}[\widetilde{P}]\left(T=T_{v} \mid a_{1}, a_{2}\right) \\
& \cdot \sum_{b_{11}, b_{12}, b_{13}}[\widetilde{P}]\left(a_{1} \mid b_{11}, b_{12}, b_{13}\right) \times[\widetilde{P}]\left(b_{11}\right) \times[\widetilde{P}]\left(b_{12}\right) \\
& \times[\widetilde{P}]\left(b_{13}\right) . \sum_{b_{21}, b_{22}, b_{23}}[\widetilde{P}]\left(a_{2} \mid b_{21}, b_{22}, b_{23}\right) \times[\widetilde{P}]\left(b_{21}\right) \\
& \times[\widetilde{P}]\left(b_{22}\right) \times[\widetilde{P}]\left(b_{23}\right) .
\end{aligned}
$$

中间节点 $A_{1}$ 和 $A_{2}$ 的区间值三角模糊边缘概率分布 如表3所示.

由式(31)可得整个传动系统的区间值模糊概率分 布如表4所示. 表4中叶节点 $X$ 处于正常状态时的三角

\begin{tabular}{|c|c|c|}
\hline 根节点 & 状态 & 状态区间值模糊概率 \\
\hline \multirow{3}{*}{$B_{i 1}$} & 0 & $\begin{array}{c}{[[0.9991,0.9994], 0.9995,} \\
[0.9996,0.9998]]\end{array}$ \\
\hline & 1 & {$[[1.7,1.9], 2,[2.2,2.4]] \times 10^{-4}$} \\
\hline & 2 & {$[[2.5,2.9], 3,[3.2,3.6]] \times 10^{-4}$} \\
\hline \multirow{3}{*}{$B_{i 2}$} & 0 & $\begin{array}{c}{[[0.9989,0.9992], 0.9993,} \\
[0.9994,0.9997]]\end{array}$ \\
\hline & 1 & - \\
\hline & 2 & {$[[6.3,6.8], 7,[7.3,8.0]] \times 10^{-4}$} \\
\hline \multirow{3}{*}{$B_{i 3}$} & 0 & $\begin{array}{c}{[[0.9986,0.9989], 0.9990,} \\
[0.9991,0.9995]]\end{array}$ \\
\hline & 1 & {$[[3.1,3.7], 4,[4.3,4.6]] \times 10^{-4}$} \\
\hline & 2 & {$[[5.4,5.8], 6,[6.4,6.9]] \times 10^{-4}$} \\
\hline
\end{tabular}
模糊概率值的上界区间大于 1 , 此时需要运用 2.3 节的 区间值三角模糊概率归一化方法来处理, 系统最终的 (归一化的)区间值模糊概率分布如表4所示.

当不考虑系统中存在的模糊不确定性时, 取根节
表 2 根节点的区间三角模糊先验概率分布 Table 2 The interval triangular fuzzy prior probability distribution of root nodes

表 $3 A_{1}$ 与 $A_{2}$ 的区间值三角模糊边缘概率分布

Table 3 The interval triangular fuzzy marginal probability distribution of nodes $A_{1}$ and $A_{2}$

\begin{tabular}{cc}
\hline 状态 & 节点 $A_{1}, A_{2}$ 状态区间值模糊概率 \\
\hline \multirow{2}{*}{0} & {$[[0.996604,0.997502], 0.997802,[0.998101$,} \\
& $0.999000]]$ \\
1 & {$[[4.790082,5.591916], 5.992602$,} \\
& $[6.493348,6.996884]] \times 10^{-4}$ \\
2 & {$[[1.417951,1.548839], 1.599190$,} \\
& $[1.689590,1.850787]] \times 10^{-3}$ \\
\hline
\end{tabular}

表 4 传动系统的区间值模糊概率分布

Table 4 The interval triangular fuzzy probability distribution of transmission system

\begin{tabular}{ccc}
\hline 状态 & $\begin{array}{c}\text { 节点 } X \text { 的状态区间值 } \\
\text { 模糊概率 }\end{array}$ & $\begin{array}{c}\text { 归一化的节点 } X \text { 状态区 } \\
\text { 间值模糊概率 }\end{array}$ \\
\hline \multirow{4}{*}{0} & {$[[0.997000,0.999216]$,} & {$[[0.999979,0.999994]$,} \\
& 0.999995, & $0.9999950,[0.999997,1]]$ \\
& $[1.000875,1.003097]]$ & \\
1 & {$[[1.587869,2.044891]$,} & {$[[2.271995,2.275415]$,} \\
& 2.275775, & 2.275775, \\
& $[2.615854,3.079512]] \times 10^{-6}$ & $[2.276305,2.280191]] \times 10^{-6}$ \\
2 & {$[[2.010585,2.398903]$,} & {$[[2.554405,2.557162]$,} \\
& 2.557409, & 2.557409, \\
& $[2.854713,3.425412]] \times 10^{-6}$ & $[2.557872,2.562178]] \times 10^{-6}$ \\
\hline
\end{tabular}

点概率区间值三角模糊数的中间值作为各节点状态 的精确概率值. 运用MATLAB及BNT工具箱计算中间 
节点 $A_{1}$ 和 $A_{2}$ 的边缘概率分布及系统叶节点 $X$ 处于各个 状态的概率, 结果如表 5 和 6 所示.

经对比, 表 5,6分别与表 3, 4中的计算结果相吻合, 进而验证了区间值三角模糊数运算的正确性, 同时也 说明了可应用区间值三角模糊数来表征系统状态的 模糊性和不确定性. 应用式(14)和(15)对表4中归一化 的系统状态区间值模糊概率进行处理, 得到系统处于 状态 0,1 和 2 的概率均值区间为

$\left\{\begin{array}{l}M([\widetilde{P}](x=0))=[0.999992,0.999996], \\ M([\widetilde{P}](x=1))=[2.275085,2.276599] \times 10^{-6}, \\ M([\widetilde{P}](x=2))=[2.556867,2.558281] \times 10^{-6} .\end{array}\right.$

\section{3 考虑 CCF 时基于区间值模糊多态 $\mathrm{BN}$ 的系统可 靠性分析}

对于可靠性要求极高的系统, 如航空航天系统、 核动力系统等, 相关失效是该类系统中机械部件的一 类不容忽视的失效形式. 传统系统可靠性分析方法往 往假设系统各部分的失效是独立的, 或为了简化分析 过程直接忽略系统失效的相关性, 这将导致系统的可 靠性分析结果存在较大误差 ${ }^{[3,35-38]}$.

假定传动系统中所有部件的寿命服从指数分布, 若将系统不完全失效状态严格归类为失效状态时, 根 据式(25)可计算出 $A_{1}$ 和 $A_{2}$ 的区间值模糊失效率为

$$
\begin{aligned}
{[\tilde{\lambda}]_{1}=} & {[\tilde{\lambda}]_{2}=[[3.335001,6.336018],} \\
& 7.334730,[8.337084,11.33926]] \times 10^{-7} .
\end{aligned}
$$

由于卫星天线双轴定位机构的运行环境非常复 杂, 故其共因失效因子应取一个较大值, 本节取传动

表 5 不考虑模糊不确定性时 $A_{1}$ 与 $A_{2}$ 的边缘概率分布 Table 5 The marginal probability distribution of nodes $A_{1}$ and $A_{2}$ without considering fuzzy uncertainty

\begin{tabular}{cccc}
\hline$A_{1}, A_{2}$ 状态 & 2 & 1 & 0 \\
\hline 状态概率 & $1.599190 \times 10^{-3}$ & $5.992602 \times 10^{-4}$ & 0.997802 \\
\hline
\end{tabular}

表 6 不考虑模糊不确定性时传动系统的概率分布

Table 6 The probability distribution of transmission system without considering fuzzy uncertainty

\begin{tabular}{cccc}
\hline$X$ 状态 & 2 & 1 & 0 \\
\hline 状态概率 & $2.557409 \times 10^{-6}$ & $2.275775 \times 10^{-6}$ & 0.999995 \\
\hline
\end{tabular}

系统的共因失效因子为 $10 \%$. 根据式(29), 共因失效事 件的区间值三角模糊失效率为

$\left[\tilde{\lambda}_{c}\right]=[[3.703909,7.034076], 8.141735$,

$$
[9.253136,12.58015]] \times 10^{-8},
$$

从而可计算得到 $t=3000 \mathrm{~h}$ 时共因节点的概率分布, 如表7所示.

基于本文 3.2 节和 3.3 节提出的贝叶斯网络构建 方法, 得到共因失效下传动系统的贝叶斯网络模型 如图7所示. 由于共因节点 $C$ 只考虑两个状态, 中间节 点 $A_{1}$ 和 $A_{2}$ 分别具有 3 个状态, 则可得到下一层中间节点 $D_{1}, D_{2}$ 的条件概率分布如表 8 所示. 由于节点 $D_{1}, D_{2}$ 均 为三状态节点, 因此在考虑共因失效时叶节点 $X$ 的条 件概率分布表与表 1 相同.

从而得到系统可靠度的表达式为

$$
\begin{aligned}
& {\left[\widetilde{p}^{c}\right](x=0) } \\
= & \sum[\widetilde{p}]\left(b_{11}, b_{12}, b_{13}, a_{1}, d_{1}, b_{21}, b_{22}, b_{23}, a_{2}, d_{2}, c, x\right) \\
= & \sum_{d_{1}, d_{2}}\left\{[\widetilde{p}]\left(x=0 \mid d_{1}, d_{2}\right)\right) \cdot \sum_{a_{1}, c}[\widetilde{p}]\left(d_{1} \mid a_{1}, c\right)[\widetilde{p}](c)
\end{aligned}
$$

表 7 共因失效节点 $C$ 的概率分布

Table 7 The probability distribution of CCF node $C$

\begin{tabular}{cc}
\hline 状态 & 节点 $C$ 区间值三角模糊概率分布 \\
\hline \multirow{2}{*}{0} & {$[[0.999622,0.999722], 0.999756$,} \\
& $[0.999789,0.999889]]$ \\
2 & {$[[1.111111,2.110000], 2.442222$,} \\
& $[2.775556,3773333]] \times 10^{-4}$ \\
\hline
\end{tabular}

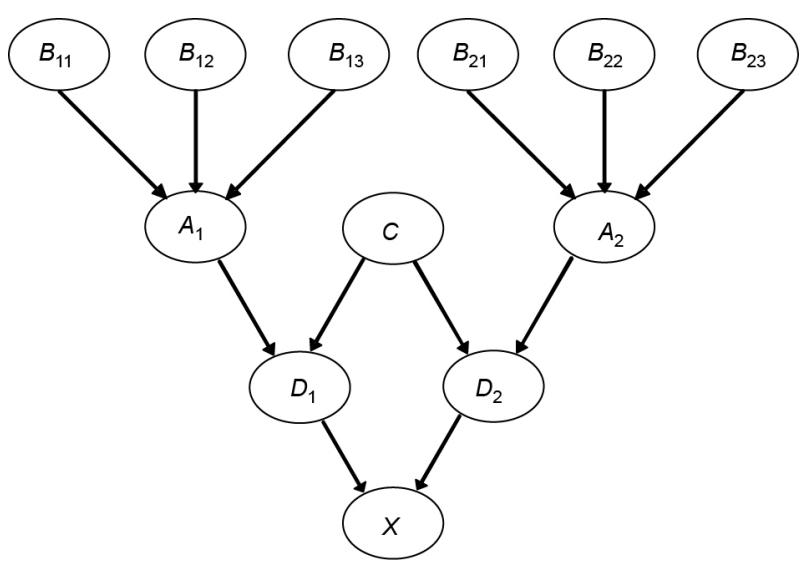

图 7 考虑共因失效的传动系统贝叶斯网络模型

Figure 7 The BN model of transmission system when considering common cause failure. 
表 8 节点 $D_{1}$ 与 $D_{2}$ 的条件概率表

Table 8 The conditional probability table of node $D_{1}$ and $D_{2}$

\begin{tabular}{ccccc}
\hline$a_{1}, a_{2}$ & $c$ & \multicolumn{3}{c}{$d_{1}, d_{2}$} \\
\cline { 3 - 5 } & & 0 & 1 & 2 \\
\hline 0 & 0 & 1 & 0 & 0 \\
0 & 2 & 0 & 0 & 1 \\
1 & 0 & 0 & 1 & 0 \\
1 & 2 & 0 & 0 & 1 \\
2 & 0 & 0 & 0 & 1 \\
2 & 2 & 0 & 0 & 1 \\
\hline
\end{tabular}

$\cdot \sum_{b_{11}, b_{12}, b_{13}}\left[[\widetilde{p}]\left(a_{1} \mid b_{11}, b_{12}, b_{13}\right)[\widetilde{p}]\left(b_{11}\right)\right.$

$\left.\cdot[\widetilde{p}]\left(b_{12}\right)[\widetilde{p}]\left(b_{13}\right)\right]$

$\sum_{a_{2}, c}[\widetilde{p}]\left(d_{2} \mid a_{2}, c\right)[\widetilde{p}](c)$

$\cdot \sum_{b_{21}, b_{22}, b_{23}}\left[[\widetilde{p}]\left(a_{2} \mid b_{21}, b_{22}, b_{23}\right)[\tilde{p}]\left(b_{21}\right)\right.$

$\left.\left.\cdot[\widetilde{p}]\left(b_{22}\right)[\widetilde{p}]\left(b_{23}\right)\right]\right\}$.

由式(30)和(35) 得到共因失效下中间节点 $D_{1}$ 和 $D_{2}$ 以 及系统 $X$ 的区间值三角模糊概率分布分别如表 9 和 10 所 示

对表 10 中归一化后的系统状态区间值三角模糊 概率进行处理, 由式(14)和(15)计算得到共因失效下系 统处于状态 0,1 和 2 的概率均值区间分别为

$$
\left\{\begin{array}{l}
M\left(\left[\widetilde{P}^{c}\right](x=0)\right)=[0.999991,0.999996], \\
M\left(\left[\widetilde{P}^{c}\right](x=1)\right)=[2.566431,2.568347] \times 10^{-6}, \\
M\left(\left[\widetilde{P}^{c}\right](x=2)\right)=[3.395690,3.398277] \times 10^{-6} .
\end{array}\right.
$$

比较表 4 和 10 , 共因失效下传动系统处于状态 0 的 概率(见表10)要明显大于不考虑共因失效时的概率值 (见表4). 这表明共因失效对卫星天线双轴定位机构传

表 9 中间节点 $D_{1}$ 与 $D_{2}$ 的区间值模糊概率分布

Table 9 The interval triangular fuzzy probability distribution of nodes $D_{1}$ and $D_{2}$

\begin{tabular}{cc}
\hline 状态 & 节点 $D_{1}, D_{2}$ 状态区间值模糊概率 \\
\hline 0 & {$[[0.996227,0.997225], 0.997558,[0.997891,0.998889]]$} \\
& {$[[4.788271,5.590361], 5.991140$,} \\
& $[6.491978,6.996108]] \times 10^{-4}$ \\
2 & {$[[1.528360,1.759326], 1.843022$,} \\
& $[1.966910,2.228500]] \times 10^{-3}$ \\
\hline
\end{tabular}

表 10 考虑共因失效时传动系统的区间值模糊概率分布 Table 10 The interval triangular fuzzy probability distribution of transmission system when considering CCF

\begin{tabular}{ccc}
\hline $\begin{array}{c}\text { 状 } \\
\text { 态 }\end{array}$ & $\begin{array}{c}\text { 叶节点 } X \text { 状态区间值 } \\
\text { 模糊概率 }\end{array}$ & $\begin{array}{c}\text { 归一化的叶节点 } X \text { 状态 } \\
\text { 区间值模糊概率 }\end{array}$ \\
\hline & {$[[0.997000,0.999216]$,} & \\
0 & 0.999995, & \\
& {$[[0.999975,0.999993]$,} \\
& $[1.000875,1.003097]]$ & $0.9999945,[0.999996,1]]$ \\
1 & {$[[1.587869,2.044891]$,} & {$[[2.562530,2.566861]$,} \\
& $[2.615854,3.079512]] \times 10^{-6}$ & $[2.567918,2.572971]] \times 10^{-6}$ \\
& {$[[2.010585,2.398903]$,} & {$[[3.390945,3.396273]$,} \\
2 & 2.557409, & 3.396731, \\
& $[2.854713,3.425412]] \times 10^{-6}$ & $[3.397447,3.405289]] \times 10^{-6}$ \\
\hline
\end{tabular}

动系统的可靠性有显著影响,在设计中有必要采取措 施来避免或减少共因失效对系统的影响.

\section{5 结论}

本文在传统贝叶斯网络和模糊理论基础上, 定义 了区间值模糊多态贝叶斯网络, 提出区间值模糊数的 归一化处理方法. 以共因失效量化的常用模型 $\beta$ 因子 参数模型)为基础, 将系统中存在的共因失效以新增独 立节点的方式融入贝叶斯网络建模过程中. 并运用基 于区间值模糊多态贝叶斯网络对具有共因失效的多 态系统进行可靠性分析, 结果表明本文所提方法可有 效处理工程系统中由于数据缺失或认知不足造成的 模糊性, 并能够对具有共因失效问题的多态系统进行 可靠性分析.

本文方法充分利用贝叶斯网络的概率推理优势, 在不需要计算系统最小割集, 或确定系统失效概率复 杂代数表达式的情况下, 可有效表征和量化认知不确 定性及共因失效对系统可靠性的影响, 符合工程实际 需求. 因此, 本文所提方法具有较强的工程意义. 本文 仅对部件存在 3 种状态的情况进行了实例研究, 也仅 考虑俯仰轴和方向轴之间的共因失效. 然而, 本文所 提方法对具有更多状态的复杂系统及更加复杂的共 因失效情况同样适用. 贝叶斯网络可简便计算每个共 因事件的后验概率分布及每个部件的重要度. 此外, 本文的多态系统定性和定量评估方法也可对系统故 障诊断和维修策略的制定提供理论依据. 


\section{参考文献}

1 Xiao N C, Huang H Z, Wang Z, et al. Reliability analysis of series systems with multiple failure modes under epistemic and aleatory uncertainties. Proc Inst Mech Eng Part O-J Risk Reliab, 2012, 226: 295-304

2 Huang H Z, Zhang X, Meng D B, et al. A new multidisciplinary design optimization method accounting for discrete and continuous variables under aleatory and epistemic uncertainties. Int J Comp Intell Syst, 2012, 5: 93-110

3 Zhang Z, Jiang C, Wang G G, et al. An efficient reliability analysis method for structures with epistemic uncertainty using evidence theory. In: Proceedings of ASME 2014 International Design Engineering Technical Conference and Computers and Information in Engineering Conference. New York: ASME, 2014

4 Mula J, Poler R, Garcia-Sabater J P. Material requirement planning with fuzzy constraints and fuzzy coefficients. Fuzzy Sets Syst, 2007, 158: 783-793

5 Yang X, Liu Y, Zhang Y, et al. Hybrid reliability analysis with both random and probability-box variables. Acta Mech, 2015, 226: 1341-1357

6 Sankararaman S, Mahadevan S. Likelihood-based representation of epistemic uncertainty due to sparse point data and/or interval data. Reliab Eng Syst Safety, 2011, 96: 814-824

7 Tonon F. Using random set theory to propagate epistemic uncertainty through a mechanical system. Reliab Eng Syst Safety, 2004, 85: 169-181

8 Ben-Haim Y. Uncertainty, probability and information-gaps. Reliab Eng Syst Safety, 2004, 85: 249-266

9 Wang X M, Li Y F, Li A F, et al. Reliability modelling and evaluation for rectifier feedback system based on continuous time Bayesian networks under fuzzy numbers (in Chinese). J Mech Eng, 2015, 51: 167-174 [王晓明, 李彦锋, 李爱峰, 等. 模糊数据下基于连续时间贝叶斯网络的 整流回馈系统可靠性建模与评估. 机械工程学报, 2015, 51: 167-174]

10 Soundappan P, Nikolaidis E, Haftka R T, et al. Comparison of evidence theory and Bayesian theory for uncertainty modeling. Reliab Eng Syst Safety, 2004, 85: 295-311

11 Yin X W, Qian W X, Xie L Y. Multi-state system reliability modelling and assessment based on Bayesian networks (in Chinese). J Mech Eng, 2009, 45: 206-212 [尹晓伟, 钱文学, 谢里阳. 基于贝叶斯网络的多状态系统可靠性建模与评估. 机械工程学报, 2009, 45: 206-212]

12 Zhou Z, Jin G, Dong D, et al. Reliability analysis of multistate systems based on Bayesian networks. In: Proceedings of 13th International Conference on Quality, Reliability, Risk, Maintenance, and Safety Engineering. Chengdu: IEEE, 2006. 344-352

13 Boudali H, Dugan J B. A discrete-time Bayesian network reliability modeling and analysis framework. Reliab Eng Syst Safety, 2005, 87: 337-349

14 Boudali H, Dugan J B. A continuous-time bayesian network reliability modeling, and analysis framework. IEEE Trans Rel, 2006, 55: 86-97

15 Vaurio J K. Availability of redundant safety systems with common-mode and undetected failures. Nucl Eng Des, 1980, 58: 415-424

16 Zhang R J, Zhang L L, Wang X W, et al. Research on reliability of multi-state system based on interval triangular fuzzy Bayesian networks (in Chinese). China Mech Eng, 2015, 8: 1092-1097 [张瑞军, 张路路, 王晓伟, 等. 区间三角模糊多态贝叶斯网络可靠性分析方法研究. 中 国机械工程, 2015, 8: 1092-1097]

17 Zhang S F. Extended TOPSIS method for dynamic interval-valued triangular fuzzy multi-attribute decision making (in Chinese). Math Pract Theory, 2013, 43: 183-187 [张市芳. 动态区间三角模糊多属性决策的TOPSIS扩展方法. 数学的实践与认识, 2013, 43: 183-187]

18 Huang Z L, Luo J. Possibility degree relation method for triangular fuzzy number-based uncertain multi-attribute decision making (in Chinese). Control Decis, 2015, 30: 1365-1371 [黄智力, 罗键. 三角模糊型不确定多指标决策的可能度关系法. 控制与决策, 2015, 30: 1365-1371]

19 Zhang X F, Zhang F X, Meng G W. Property of four arithmetic operations of fuzzy number and linear equation of fuzzy number (in Chinese). Fuzzy Syst Math, 2005, 19: 93-98 [张兴芳, 张风霞, 孟广武. 模糊数的四则运算性质及其线性方程. 模糊系统与数学, 2005, 19: 93-98]

20 Ma D Z, Zhou Z, Yu X Y, et al. Reliability analysis of multi-state Bayesian networks based on fuzzy probability (in Chinese). Syst Eng Electron, 2012, 34: 2607-2611 [马德仲, 周真, 于晓洋, 等. 基于模糊概率的多状态贝叶斯网络可靠性分析. 系统工程与电子技术, 2012, 34: 2607-2611]

21 Lu Y, Li Q M, Zhou Z P. Safety risk prediction of subway operation based on fuzzy Bayesian network (in Chinese). J Southeast Univ, 2010, 40: 1110-1114 [陆荣, 李启明, 周志鹏. 基于模糊贝叶斯网络的地铁运营安全风险预测. 东南大学学报, 2010, 40: 1110-1114]

22 Chen D N, Yao C Y. Reliability analysis of multi-state system based on fuzzy Bayesian networks and application in hydraulic system (in Chinese). J Mech Eng, 2012, 48: 175-183 [陈东宁, 姚成玉. 基于模糊贝叶斯网络的多态系统可靠性分析及在液压系统中的应用. 机械工程学报, 2012, 48: 175-183]

23 Zhang L L. Research on System Reliability Analysis and Fault Diagnosis Methods Based on Bayesian Networks (in Chinese). Dissertation for 
Master Degree. Jinan: Shandong Jianzhu University, 2015 [张路路. 贝叶斯网络系统可靠性分析及故障诊断方法研究. 硕士学位论文. 济 南: 山东建筑大学, 2015].

24 Sun L N, Song T. The distance of interval-value triangular fuzzy number (in Chinese). Sciencepaper Online, 2007, 306: 1-6 [孙丽娜, 宋涛. 区 间值三角模糊数的距离. 中国科技论文在线, 2007, 306: 1-6]

25 Yao R P, Shen H Z. The multi-attribute group decision making method based on interval valued triangular fuzzy number (in Chinese). Math Pract Theory, 2015, 45: 197-203 [要瑞璞, 沈惠璋. 基于区间值三角模糊数的多属性群决策方法. 数学的实践与认识, 2015, 45: 197-203]

26 Sun H L, Yao W X. Comments on methods for ranking interval numbers (in Chinese). J Syst Eng, 2010, 25: 304-312 [孙海龙, 姚卫星. 区间 数排序方法评述. 系统工程学报, 2010, 25: 304-312] Nakahara Y, Sasaki M, Gen M. On the linear programming problems with interval coefficients. Comp Industrial Eng, 1992, 23: 301-304 Yao C Y, Chen D N, Wang B. Fuzzy reliability assessment method based on T-S fault tree and Bayesian network (in Chinese). J Mech Eng, 2014, 50: 193-201 [姚成玉, 陈东宁, 王斌. 基于T-S故障树的贝叶斯网络的模糊可靠性评估方法. 机械工程学报, 2014, 50: 193-201]

29 Liang X Y, Wu S H, Shi Q X. Study on normalization in fuzzy causality diagram (in Chinese). Microelectron Comput, 2006, 23: 1-3 [梁新元, 吴淑皇, 石庆喜. 模糊因果图的归一化研究. 微电子学与计算机, 2006, 23: 1-3]

30 Ericson C A. Hazard Analysis Techniques for System Safety. Canada: John Wiley \& Sons, 2005

31 Yin X. Common cause failure model of system reliability based on Bayesian networks. Int J Performab Eng, 2010, 6: 255-268

32 Yin X W, Qian W X, Xie L Y. Common cause failure model of system reliability based on Bayesian networks (in Chinese). China Mech Eng, 2009, 20: 90-94 [尹晓伟, 钱文学, 谢里阳. 基于贝叶斯网络的系统可靠性共因失效模型. 中国机械工程, 2009, 20: 90-94]

33 Börcsök J, Schaefer S. Estimation and evaluation of common cause failures. In: Proceedings of Second International Conference on Systems. Martinique: IEEE, 2007. 867-882

34 Mi J, Li Y F, Huang H Z, et al. Reliability analysis of multi-state system with common cause failure based on Bayesian networks. Eksploat Niezawodn, 2013, 15: 169-175

35 Jin N. Multi-State Reliability Analysis of a Large Deployable Satellite Antenna Based on Bayesian Networks (in Chinese). Dissertation for Master Degree. Xi'an: Xidian University, 2011 [靳宁. 基于贝叶斯网络的大型星载展开天线多状态可靠性分析. 硕士学位论文. 西安: 西安电 子科技大学, 2011]

36 Zhang H. The System Reliability Analysis of Dual-Axis Positioning Mechanism for Space-Born Antenna (in Chinese). Dissertation for Master Degree. Chengdu: University of Electronic Science and Technology of China, 2011 [张华. 星载天线双轴定位机构的系统可靠性分析. 硕士 学位论文. 成都: 电子科技大学, 2011]

37 Liao Y, Li C J, Feng X J, et al. Simulation and verification to the virtual prototype of two-axis-position structure based on Pro/E (in Chinese). Aerosp Shanghai, 2006, 23: 59-65 [廖瑛, 李长江, 冯向军, 等. 基于Pro/E的双轴定位机构虚拟样机的建模与校核. 上海航天, 2006, 23: 59-65]

38 Braband J, Vom Hövel R, Schäbe H. Probability of failure on demand-the why and the how. In: Computer Safety, Reliability, and Security. Berlin: Springer, 2009 


\title{
Reliability modeling and analysis of complex multi-state system based on interval fuzzy Bayesian network
}

\author{
MI JinHua, LI YanFeng*, PENG WeiWen \& HUANG HongZhong \\ Center for System Reliability and Safety, University of Electronic Science and Technology of China, Chengdu 611731, China
}

With the increasing complexity and large size of modern advanced engineering systems, the traditional reliability analysis and evaluation technology which is based on large number of sample data cannot meet the demand of complex system. Aiming at the engineering application requirement, this paper focuses on the reliability modeling and analysis of complex system with uncertainties and failure dependencies. Due to the diversity of input information and the system failure factors, and system redundancies, the uncertainty and common cause failure (CCF) have become the most important factors for reliability analysis and evaluation of complex system. In consideration of the epistemic uncertainty caused by lack of probability statistical information, the fuzzy theory is employed to express the fuzzy information of system, and the basic events failure probabilities are described by interval-valued fuzzy numbers. Taking account of the influence of CCF to system reliability and the widespread presence of MSS in engineering practices, the CCF is quantified by the $\beta$ factor parameter model and integrated to Bayesian Network (BN) model through a new defined common cause node. Finally, a comprehensive method for reliability modeling and assessment of a multi-state system (MSS) with CCFs based on interval-valued fuzzy BN is proposed by taking the advantage of graphic representation and uncertainty reasoning of BN. The method has applied to the transmission system of two-axis positioning mechanism of a satellite antenna to demonstrate its effectiveness and capability for directly calculating the system reliability on the basis of multi-state probabilities of components. It has shown that the method proposed has done further improvement of the theory for reliability analysis of complex system and can realize its engineering application.

epistemic uncertainty, multi-state system, interval fuzzy probability, Bayesian network, common cause failure, reliability modelling

PACS: 47.27.-i, 47.27.Eq, 47.27.Nz, 47.40.Ki, 47.85.Gj

doi: $10.1360 /$ SSPMA2016-00521 